\title{
A PASSIVE DYNAMIC APPROACH FOR FLAPPING-WING MICRO-AERIAL VEHICLE CONTROL
}

\author{
Katie Byl \\ Robotics Lab \\ Department of Electrical and Computer Engineering \\ University of California \\ Santa Barbara, California 93106 \\ Email: katiebyl@ece.ucsb.edu
}

\begin{abstract}
This article outlines a new control approach for flapping-wing micro-aerial vehicles (MAVs), inspired both by biological systems and by the need for lightweight actuation and control solutions. In our approach, the aerodynamic forces required for agile motions are achieved indirectly, by modifying passive impedance properties that couple motion of the power stroke to the angle of attack (AoA) of the wing. This strategy is theoretically appealing because it can exploit an invariant, cyclical power stroke, for efficiency, and because an impedance-adjusting strategy should also require lower bandwidth, weight, and power than direct, intra-wingbeat control of AoA. We examine the theoretical range of control torques and forces that can be achieved using this method and conclude that it is a plausible method of control. Our results demonstrate the potential of a passive dynamic design and control approach in reducing mechanical complexity, weight and power consumption of an MAV while achieving the aerodynamic forces required for the types of high-fidelity maneuvers that drive current interest in autonomous, flapping-wing robotics.
\end{abstract}

\section{NOMENCLATURE}

$c(r)$ Chord length of wing, at radius $r$ $c_{m}(r)$ Mean chord position, at radius $r$

$C O P$ Center of pressure on wing during flapping

$\bar{c}_{w} \quad$ Normalized mean chord length
$C_{D} \quad$ Drag coefficient

$C_{L} \quad$ Lift coefficient

$C_{N} \quad$ Wing-normal force coefficient

$C_{T} \quad$ Wing-tangential force coefficient

$f_{D} \quad$ Drag force on a blade element

$F_{D} \quad$ Drag force on the entire wing

$f_{L} \quad$ Lift force on a blade element

$F_{L} \quad$ Lift force on the entire wing

$F_{L}^{*} \quad$ Lift force when $\alpha_{r}$ is optimal

$f_{w} \quad$ Flapping frequency $(\mathrm{Hz})$

$F_{N} \quad$ Aerodynamic force normal to wing

$F_{T} \quad$ Aerodynamic force tangential to wing

$k_{w} \quad$ Spring coefficient for wing rotation

$\hat{k}_{w} \quad$ Non-dimensionalized spring coef.

$m \quad$ Mass of the MAV

$\hat{r}_{\text {cop }} \quad$ Normalized, radial distance to COP on wing

Re Reynolds number

$R_{w} \quad$ Span of a single wing, root to tip

$S_{w} \quad$ Shape factor (non-dimensional) for the wing

$u_{r} \quad$ Air velocity, relative to wing

$\hat{z}_{\text {cop }} \quad$ Normalized, spanwise distance to COP on wing

$\alpha \quad$ Geometric angle of attack, relative to body

$\alpha_{r} \quad$ True angle of attack, relative to local airflow

$v \quad$ Kinematic viscosity

$\rho \quad$ Density of air

$\tau_{k} \quad$ Wing-pitching torque due to spring forces

$\tau_{\psi} \quad$ Wing-pitching torque due to aero forces

$\phi \quad$ Stroke angle of wing

$\psi \quad$ Pitch rotation angle of wing

$\psi_{o} \quad$ Offset angle for passive spring 
$\omega_{w} \quad$ Flapping frequency $(\mathrm{rad} / \mathrm{s})$

\section{INTRODUCTION}

Many recent approaches to robot locomotion are inspired by the astonishing agility, efficiency and/or robustness of animal locomotion [1-3]. In this paper, we suggest a new approach for the control of underactuated, flapping-wing micro-aerial vehicles (MAVs) and examine its theoretical feasibility. Our motivation is to develop a method that (1) could realistically be implemented to provide a simple, low-weight actuation solution for a real robot, (2) exploits and modifies passive dynamics to steer. Throughout this paper, we assume a difference in time scale between fast flapping and the (slower) time-averaged control forces and torques that steer agile maneuvers. Because it takes several wingbeats for an insect or robot to complete a flapping-wing maneuver, we assume we can use the time-averaged aerodynamics over the course of a wingbeat to closely approximate the true forces over time.

\section{Bio-Inspired Flapping Flight Control}

Insects such as flies use two types of muscles in flight [4]. One set primarily provides power, employing cyclical high frequency, low variability motion. The second set operates indirectly and functions, primarily, to generate steering forces rather than to drive the gross velocity of the wing [5]. Although flies demonstrate amazingly agile maneuvers, as anyone who has attempted to catch one can verify, they employ a "remarkable economy of control" [6] in doing so. They are most certainly underactuated, with significant coupling between the forces and torques generated to control the six degrees of freedom of the body [6]. An underactuated animal or robot, however, can still be controllable, in the sense of finding some motion control plan to orient all six degrees of freedom into a particular configuration in finite time.

We examine the feasibility of a similar, bioinspired approach, where a motor and transmission are used to drive a gross flapping motion in a harmonic motion, while the angle of attack (AoA) is indirectly determined via the impedance (stiffness, damping, and inertia) relationship between the wing spar and the wing itself. Although there is direct evidence that real insects employ active methods to flip the wing to control AoA [7], there is also evidence that passive wing pitch rotation is sufficient to generate lift and drag forces for flight [8]. We investigate a new approach where pitch rotation is controlled indirectly, through the mechanism of an adjustable impedance.

Other researchers have already considered the case of an actively-powered flapping motion with passive but unrealistically instantaneous rotation of the pitch angle, $\psi$. This idealization assumes that there is no impedance to resist aerodynamic forces and that the wing flips over instantly until it hits a "hard stop" at some prescribed AoA $[9,10]$. Such modeling approaches also assume constant velocity through the forward or backward portions of the wing stroke, $\phi$, requiring a step change in velocity as the wing reverses direction. These design assumptions are impractical if not impossible to achieve in a real flight vehicle, and they do not allow for any variations in AoA.

In this paper, we assume the wing "hinge" determining angle of attack has a finite impedance, which can be adjusted through the use of indirect control actuators. Specifically, we analyze a simplified case, where this impedance is a spring; that is, the mass of the wing and any damping at the wing hinge are both assumed to be negligible for this particular analysis. By adjusting the properties of this spring, one indirectly adjusts the relationship between stroke angle and angle of attack, and the question is: "How large are the subsequent steering forces that can be generated?"

\section{METHODS}

This section outlines the model and methods employed in quantifying the utility of this passive dynamic approach for controlling output forces and torque to maneuver the body of a small, flapping-wing vehicle. We assume that only the impedance properties of each wing may change over time, indirectly resulting in variations in the angle of attack throughout a wingbeat that result in some net force over time. In practice, this approach would best be used in conjunction with modulation of the frequency and/or waveform of the wing stroke. However, we intentionally examine an extreme case where the power stroke remains invariant, as a rigorous test of the range of aerodynamic forces that can be generated when the AoA of the wing is set "passively" by the tuned impedance, rather than being directly commanded throughout the stroke.

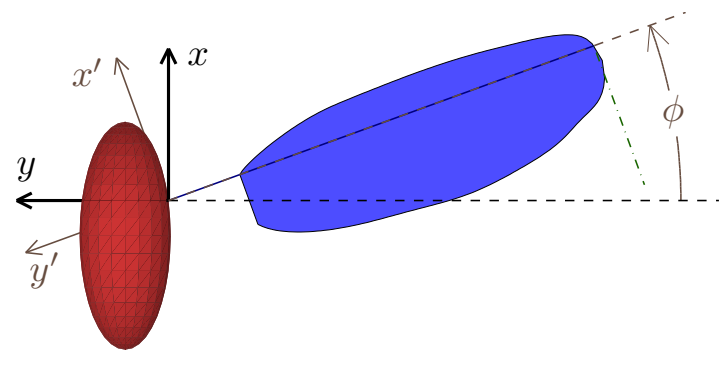

Figure 1. OVERHEAD VIEW OF WING. 


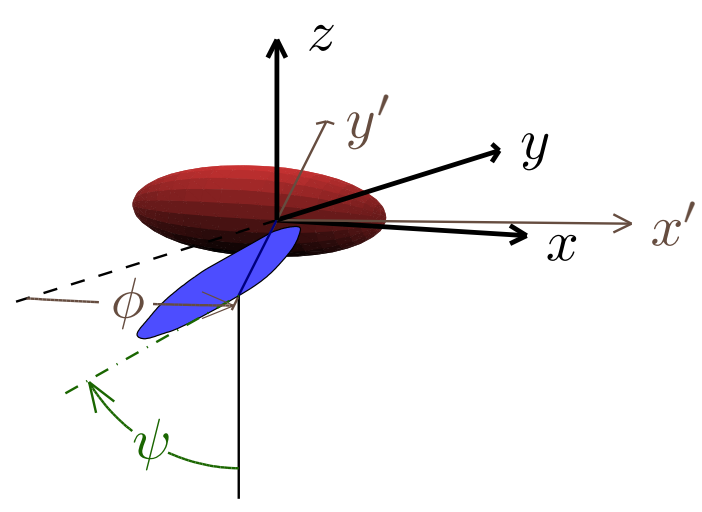

Figure 2. SIDE VIEW OF WING.

To examine this approach, we use two models, spanning a range of practical sizes that are arguably feasible for present-day flapping-wing robotics technology. Both designs employ a single wing on either side of the body, as opposed to four-wing designs such as a dragonfly. In addition to simplifying any future experimental implementations, this design is more appropriate for our modeling assumptions, since it avoids wing-wing aerodynamic interference. For the smaller of the two designs, we model a fly-scale robot based loosely on the smallest proven, lightweight flappingwing MAV design demonstrating self-sustaining lift of which we are aware to date [3], and at the large end, we scale the model roughly to the size of a hummingbird. Hummingbirds are unique among vertebrates in displaying the near-sinusoidal stroke patterns and nearsymmetrical flapping patterns typically associated with insect flight [4], and it therefore seems practical to limit our study of insect-style flapping to robotic vehicles designed at or below this size. Properties of both models are listed in Table 1, and additional details on selecting vehicles parameters are given ahead on page 6 .

\section{Aerodynamic Forces}

To model the aerodynamic forces on the wing, we employ a quasi-steady model, where the instantaneous aerodynamic forces on the wing are approximated using Blade Element Method (BEM) [11,12].

Blade Element Method (BEM). The Blade Element Method for flapping flight [13] approximates the flow across a wing element as being quasistationary. By its nature, this method neglects several unsteady effects which can contribute importantly in insect flight [14]. However, at the low to moderate Reynolds number conditions (10-20,000) [4], BEM theory is commonly used to derive a first-order estimate of the dominant forces on flapping wings typical of insect and hummingbird flight $[11,12,15,16]$.

In blade-element theory, each wing is divided into a set of cross-sectional strips, each of width $d r$, and at a mean radius $r$ from the axis of rotation of the wing on the body, as depicted in Figure 3. Although the aerodynamic effects of twisting and warping of the wings can be measurable in insect-scale flapping [17], we model each wing as a rigid, thin plate. We also ignore any inertial forces due to added mass and the mass distribution of the wing itself, which typically represent a small fraction of total force in near-hovering flight [16], and we do not include second-order effects of wing pitch velocity, $\psi$, on local wing velocity. The aerodynamic lift and drag forces on a differential blade element are then modeled as:

$$
\begin{aligned}
& f_{L}(r)=\frac{\rho}{2} c(r) u_{r}^{2} \cdot C_{L}\left(\alpha_{r}\right) \\
& f_{D}(r)=\frac{\rho}{2} c(r) u_{r}^{2} \cdot C_{D}\left(\alpha_{r}\right)
\end{aligned}
$$

where $c(r)$ is the chord length which is a function of the radius $r$ on the wing, and the relative velocity during near-hover maneuvers is approximately:

$$
u_{r}=r \dot{\phi}
$$

Integrating over the entire span, $R_{w}$, of the wing, the total lift and drag forces are then:

$$
\begin{aligned}
F_{L} & =\int_{r=0}^{R_{w}}\left(\frac{\rho}{2} c(r)[r \dot{\phi}]^{2} \cdot C_{L}\left(\alpha_{r}\right)\right) d r \\
& =C_{L}\left(\alpha_{r}\right) \cdot \frac{\rho}{2} \dot{\phi}^{2} \cdot \int_{r=0}^{R_{w}}\left(c(r) r^{2}\right) d r \\
F_{D} & =\int_{r=0}^{R_{w}}\left(\frac{\rho}{2} c(r)[r \dot{\phi}]^{2} \cdot C_{D}\left(\alpha_{r}\right)\right) d r \\
& =C_{D}\left(\alpha_{r}\right) \cdot \frac{\rho}{2} \dot{\phi}^{2} \cdot \int_{r=0}^{R_{w}}\left(c(r) r^{2}\right) d r
\end{aligned}
$$

These expressions can be written as a constant, wing-dependent shape factor (in parentheses at left in Equations 6-7, below) times a term that varies with both wing stroke speed, $\dot{\phi}$, and with angle of attack, $\alpha_{r}$ (in parentheses at right). Note that for a rectangular wing of constant chord length, $c(r)=C$, they would become:

$$
\begin{aligned}
F_{L-\text { rect }} & =\left(\frac{\rho}{2} C R_{w}^{3}\right) \cdot\left(C_{L}\left(\alpha_{r}\right) \dot{\phi}^{2}\right) \\
F_{D-r e c t} & =\left(\frac{\rho}{2} C R_{w}^{3}\right) \cdot\left(C_{D}\left(\alpha_{r}\right) \dot{\phi}^{2}\right)
\end{aligned}
$$




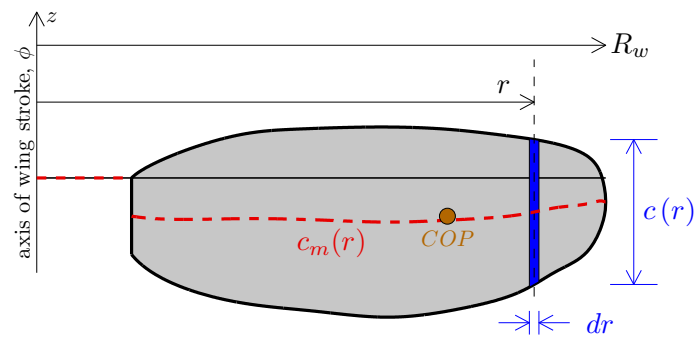

Figure 3. A BLADE ELEMENT OF THE WING.

For the arbitrary wing shape we use, shown in Figure 3, and using $\rho=1.28\left(\mathrm{~kg} / \mathrm{m}^{2}\right)$ as the density of air, the total lift and drag on a wing scale as $R_{w}^{4}$ and are:

$$
\begin{aligned}
& F_{L}=\left(0.0566 \cdot R_{w}^{4}\right) \cdot\left(C_{L}\left(\alpha_{r}\right) \dot{\phi}^{2}\right) \\
& F_{D}=\left(0.0566 \cdot R_{w}^{4}\right) \cdot\left(C_{D}\left(\alpha_{r}\right) \dot{\phi}^{2}\right)
\end{aligned}
$$

These final expressions depend on the velocity of the stroke angle, $\dot{\phi}$, and on lift and drag coefficients that vary with angle of attack. The lift and draft coefficients we use are detailed below in the next section.

To find the center of pressure (COP) on the wing, we integrate to find the torque about each rotational degree of freedom, $\phi$ and $\psi$, then divide by the force to solve for the length of the moment arm. Note that because lift and drag forces are related by the same ratio for any location on the wing, we can use either 8 or ?? as a proxy to solve for the COP, calculating only the component of torque due to that particular force. Solving for the torques due to lift, we obtain:

$$
\begin{aligned}
\tau_{L, \phi} & =\int_{r=0}^{R_{w}}\left(r \cdot \frac{\rho}{2} c(r)[r \dot{\phi}]^{2} C_{L}\left(\alpha_{r}\right)\right) d r \\
& =\frac{\rho}{2} C_{L}\left(\alpha_{r}\right) \dot{\phi}^{2} \int_{r=0}^{R_{w}}\left(c(r) r^{3}\right) d r \\
\tau_{L, \Psi} & =\int_{r=0}^{R_{w}}\left(c_{m}(r) \cdot \frac{\rho}{2} c(r)[r \dot{\phi}]^{2} C_{L}\left(\alpha_{r}\right)\right) d r \\
& =\frac{\rho}{2} C_{L}\left(\alpha_{r}\right) \dot{\phi}^{2} \int_{r=0}^{R_{w}}\left(c_{m}(r) c(r) r^{2}\right) d r
\end{aligned}
$$

where $c_{m}(r)$ is the location of the middle of the chord at radius $r$. For our particular wing shape, the nondimensional COP location (scaled by $R_{w}$ ) is:

$$
\begin{aligned}
& \hat{r}_{\text {cop }}=0.8662 \\
& \hat{z}_{\text {cop }}=-0.0807
\end{aligned}
$$

Lift and Drag Coefficients. Experimental data for 3D insect-scale wing flapping provide the following fitted expressions for the translational lift and drag coefficients as a function of angle of attack [14]:

$$
\begin{aligned}
& C_{L-\exp }=0.225+1.58 \sin \left(2.13 \alpha_{r}-7.20^{\circ}\right) \\
& C_{D-\exp }=1.92-1.55 \cos \left(2.04 \alpha_{r}-9.82^{\circ}\right)
\end{aligned}
$$

These relationships are now widely used to approximate the lift and drag forces on MAV-scale flapping wing models [18-20], and they correspond to aerodynamic forces that are primarily directed normal to the wing, indicating viscous drag effects are far less important than pressure forces. For our simple model, we have approximated these equations with a purely normal force. These simplified curves also ensure both smoothness and symmetry (as theoretically required) near $\alpha_{r}=0^{1}$ :

$$
\begin{aligned}
C_{L} & =1.8 \sin \left(2 \alpha_{r}\right) \\
C_{D} & =1.8\left(1-\cos \left(2 \alpha_{r}\right)\right)
\end{aligned}
$$

Figure 4 shows these coefficients as a function of $\alpha_{r}$, the true angle of attack of the wing, with respect to relative air flow. Our simulation results are nearly identical, quantitatively, when we use either set of expressions, so we have adopted the simplified expressions in Equations 16 and 17 primarily to aid the reader in understanding the basic, theoretical relationships between the passive dynamics and output control forces more intuitively.

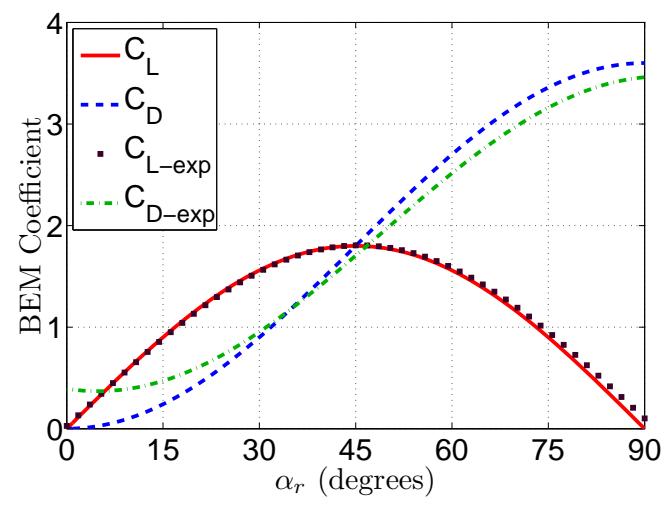

Figure 4. LIFT AND DRAG COEFFICIENTS VS ALPHA.

\footnotetext{
${ }^{1}$ By symmetry, both $C_{L}$ and the derivative of $C_{D}$ should mathematically be zero at $\alpha_{r}=0$. These requirements are not satisfied by the fit curves from experimental data, which is one reason we have chosen to adjust these curves slightly for our model.
} 
We can equivalently represent these two orthogonal force components in terms of normal and tangential force coefficients, $C_{N}$ and $C_{T}$, with respect to the local axis of the wing. From Figure 5, it is clear that the overall force is largely normal to the wing, and for our approximate relationships in Equations 16 and 17, the tangential component is identically zero, while the normal component varies as the sine of $\alpha_{r}$ :

$$
\begin{aligned}
& C_{T}=0 \\
& C_{N}=3.6 \sin \left(\alpha_{r}\right)
\end{aligned}
$$

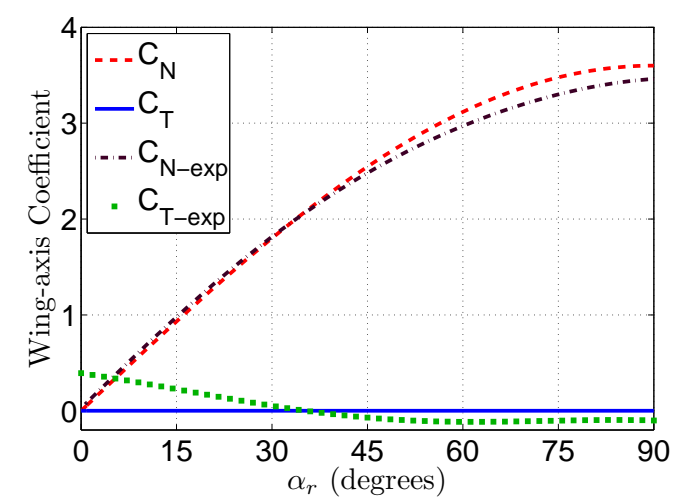

Figure 5. NORMAL AND TANGENTIAL FORCE COEFFS.

Figure 6 shows the aerodynamic forces acting on a cross-section of the wing. Here, $u_{r}$ is the direction of the flow of air, relative to a wing-fixed coordinate system, and $\alpha_{r}$ is the angle of attack, with respect to $u_{r}$. Note that we define the angle of rotation of the wing, $\psi$, with respect to a wing-fixed coordinate system, so that the geometric angle of attack, $\alpha=\frac{\pi}{2}-\psi$, is not necessarily equivalent to $\alpha_{r}$ in general, although they will be equivalent for our particular study, which examines motions initiating from a steady state of hovering.

\section{Indirect Control of Wing Pitch}

This section derives equations of motion for two indirect methods to control the wing pitch angle, $\psi$, through direct control of the stiffness, $k_{w}$ and neutral set point, $\psi_{o}$ of the wing-hinge spring. In the end, our goal is not to dictate a particular trajectory, $\psi(t)$, over time but rather to obtain time-averaged forces and torque to accelerate and steer a robotic vehicle.

Force Balance for Wing Pitch. Combining Equations 8 and 9 with our approximation from Equa-

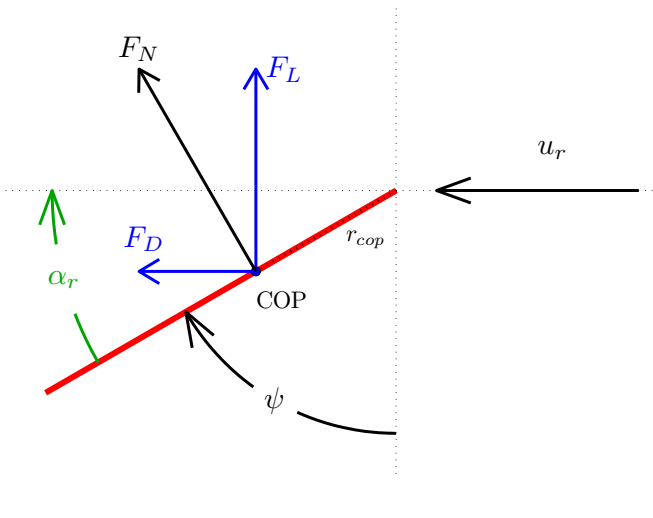

Figure 6. AERODYNAMIC FORCES ON THE WING.

tion 19 of the net force as being normal to the wing, we can solve for the net force on our wing shape as:

$$
\begin{aligned}
F_{N} & =0.0566 \cdot R_{w}^{4} \cdot\left(C_{N}\left(\alpha_{r}\right) \dot{\phi}|\dot{\phi}|\right) \\
& =0.0566 \cdot R_{w}^{4} \cdot\left(3.6 \sin \left(\alpha_{r}\right) \dot{\phi}|\dot{\phi}|\right) \\
& =0.2038 \cdot R_{w}^{4} \cdot \sin \left(\alpha_{r}\right) \cdot \dot{\phi}|\dot{\phi}|
\end{aligned}
$$

From 12 and 13, the COP at which this force acts remains at a constant position on the wing:

$$
\begin{aligned}
& r_{\text {cop }}=\hat{r}_{\text {cop }} \cdot R_{w}=0.8662 R_{w} \\
& z_{\text {cop }}=\hat{z}_{\text {cop }} \cdot R_{w}=-0.0807 R_{w}
\end{aligned}
$$

Therefore, we can write the torque driving wing pitch in terms of the moment arm and normal force as:

$$
\begin{aligned}
\tau_{\psi} & =-z_{c o p} \cdot F_{N, y^{+}} \\
& =0.0164 \cdot\left(R_{w}^{4}\right) \cdot\left(\sin \left(\alpha_{r}\right) \dot{\phi}|\dot{\phi}|\right)
\end{aligned}
$$

We assume the inertia of the wing is small, so that the aerodynamic force essentially remains balanced with the torsional stiffness of the wing pitch angle:

$$
\tau_{k_{r}}=-k_{r}\left(\psi-\psi_{o}\right)
$$

where both $k_{r}$ and $\psi_{o}$ are impedance properties we can control, for instance by adjusting the tensions in an opposing pair of tendons along each wing. By equating 24 and 25 , we can solve at any moment for $\psi$ as a function 
of $\phi$ and $\dot{\phi}$ :

$$
\psi=\psi_{o}+\frac{0.0164}{k_{r}} \cdot R_{w}^{4} \cdot \sin \left(\alpha_{r}\right) \dot{\phi}|\dot{\phi}|
$$

\section{Robot Vehicle Scaling}

The two sets of vehicle parameters we study, given in Table 1, span a size range from fly (Diptera) through hummingbird (Trochilidae). The smaller design is based closely on proven designs of the Harvard microrobotic fly [3], which we believe is still the smallest and lightest flapping-wing robot capable of sufficient lift generation for sustained flight. The larger design is scaled to match allometric data for hummingbirds, since they are the largest animals that employ insect-style flapping [4]. Our model of the fluid dynamics assumes a viscous Reynolds number regime. Hummingbirds typically hover at or just exceeding this range ( 5000-30,000) [21], and we have intentionally designed our bird-scale model to lie within in this range, with $R e=20,000$. Although some turbulent effects may begin to appear with a real robot flapping at these wing velocities, we believe this is a reasonable upper limit at which our model would capture first-order dynamics. To calculate the Reynolds number $(\operatorname{Re}=u L / \mathrm{v})$ for a flapping wing, we use the peak airflow velocity across the chord located at the center of pressure on the wing:

$$
R e=\frac{\left(R_{w} \hat{r}_{c o p} \cdot \phi_{\max } \cdot \omega_{w}\right) \cdot\left(R_{w} \bar{c}_{m}\right)}{v_{a i r}}
$$

For our geometry, $\hat{r}_{c o p}=0.866, \bar{c}_{m}=0.333$, and $\phi_{\max }=$ $\pi / 3$. Using $v_{\text {air }} \approx 15.11 \times 10^{-} 6\left(\mathrm{~m}^{2} / \mathrm{s}\right)$, we can write $R e$ in terms of the wing length in $\mathrm{m}, R_{w}$, and frequency in Hertz $\left(f_{w}=2 \pi \omega\right)$ as:

$$
\operatorname{Re}=\left(R_{w}^{2} \cdot f_{w}\right) \cdot 125,000\left(\mathrm{~s} / \mathrm{cm}^{2}\right)
$$

Our modeled vehicle parameters are scaled to match allometrically with agile flapping animals, to provide designs with a reasonable expectation of aerodynamic feasibility. Figures 7 and 8 illustrate how the mass, wing area and flapping frequency scale in comparison to a variety of animals (insects and hummingbirds) capable of sustained, flapping-wing hovering; these figures are adapted from Figures $3.3 \mathrm{~A}$ and $3.3 \mathrm{~B}$ in [4], with our robot parameters overlaid.

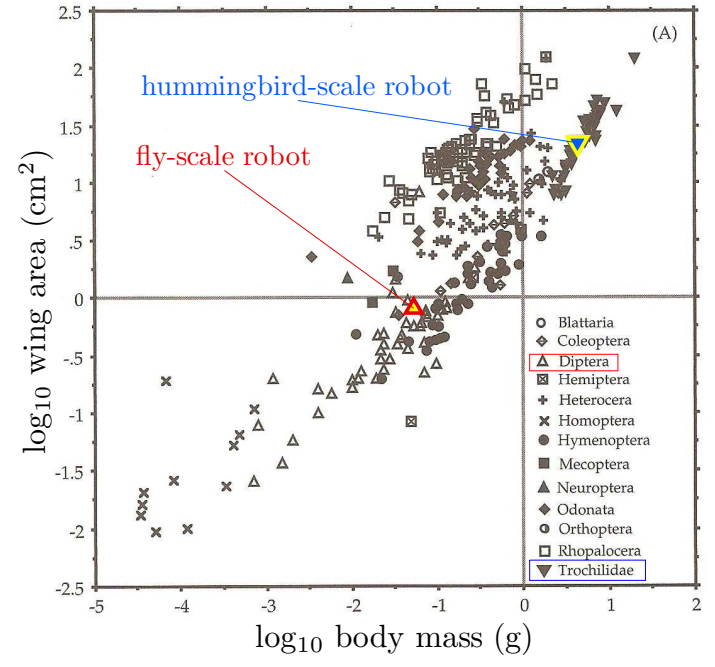

Figure 7. ALLOMETRIC DATA: WING AREA [4].

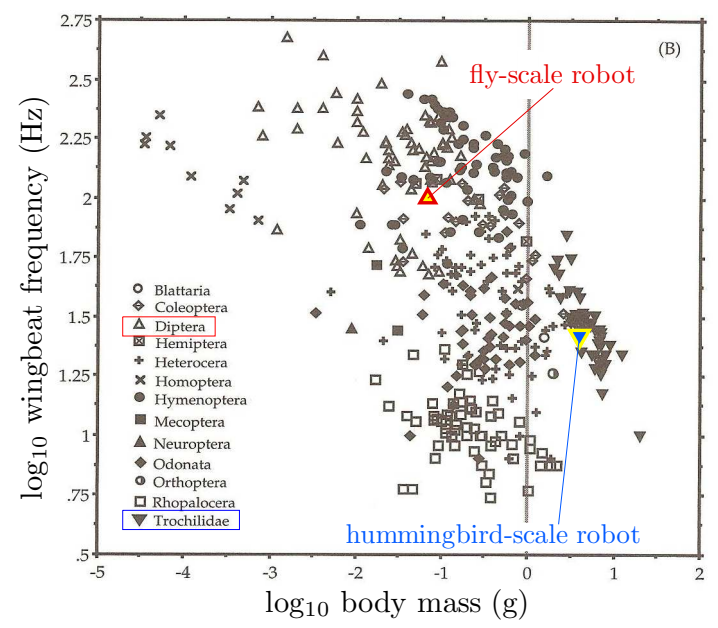

Figure 8. ALLOMETRIC DATA: FREQUENCY [4].

\section{RESULTS}

Here, we summarize our results for both fly- and hummingbird-scale flapping wing flight with tunable stiffness of the wing pitch degree of freedom. Table 1 shows the characteristics of each model along with predicted forces and corresponding maximum accelerations in $z$ (up only) and $x$ (forward or back); no sideslip is predicted in this model $(\ddot{y}=0)$. The translational accelerations correspond to $\sim 2 g$ for the smaller vehicle, and $1 / 3$ to $1 / 2$ of gravity for the larger design. Quantitative estimates for angular rates are more difficult to predict, but achievable accelerations about both the $z$ axis (yaw) and $y$ axis (roll) are clearly significant (i.e., greater than $1000^{\circ} / \mathrm{s}^{2}$ ). After initiating a maneuver from a state of hover, actual robot accelerations would typically drop over time, since air drag on the body and wings will act to slow any translational and rotational 
Table 1. THEORETICAL CONTROL OUTPUT RANGES.

\begin{tabular}{ccc}
\hline Parameter & Fly Scale & Hummingbird Scale \\
\hline$R e$ & $\sim 2,800$ & $\sim 20,000$ \\
$\omega_{f}$ & $100 \mathrm{~Hz}$ & $25 \mathrm{~Hz}$ \\
$R_{w}$ & $1.5 \mathrm{~cm}$ & $8 \mathrm{~cm}$ \\
$\bar{F}_{L, \text { max }}$ & $.0021 \mathrm{~N}$ & $0.10 \mathrm{~N}$ \\
$\bar{F}_{D, \max }$ & $.0012 \mathrm{~N}$ & $.062 \mathrm{~N}$ \\
$m$ & $70 \mathrm{mg}$ & $6,000 \mathrm{mg}$ \\
$\bar{F}_{L, \text { max }} / g_{z}$ & $210 \mathrm{mg}$ & $10,600 \mathrm{mg}$ \\
$+\ddot{z}_{\max }$ & $19.6 \mathrm{~m} / \mathrm{s}^{2}$ & $7.5 \mathrm{~m} / \mathrm{s}^{2}$ \\
$\pm \ddot{x}_{\max }$ & $17.6 \mathrm{~m} / \mathrm{s}^{2}$ & $10.4 \mathrm{~m} / \mathrm{s}^{2}$ \\
$\tau_{x, \max }$ & $1.8 \mathrm{e}-5(\mathrm{Nm})$ & $0.9 \mathrm{e}-2(\mathrm{Nm})$ \\
$\tau_{z, \max }$ & $3.1 \mathrm{e}-5(\mathrm{Nm})$ & $1.5 \mathrm{e}-2(\mathrm{Nm})$ \\
\hline
\end{tabular}

velocities significantly [22].

The remainder of this section details our simulation results. We assume flapping is maintained at a constant frequency, to isolate the capabilities of using tuned stiffness in affecting control; note that employing variable speeds and/or waveform types should certainly enhance control range beyond our presentation here. On either of the two wings, there are only two values that can be manipulated: the wing pitch stiffness $\left(k_{r}\right)$ and the null pitch angle about which the spring operates $\left(\psi_{o}\right)$.

\section{Varying the stiffness, $k_{r}$}

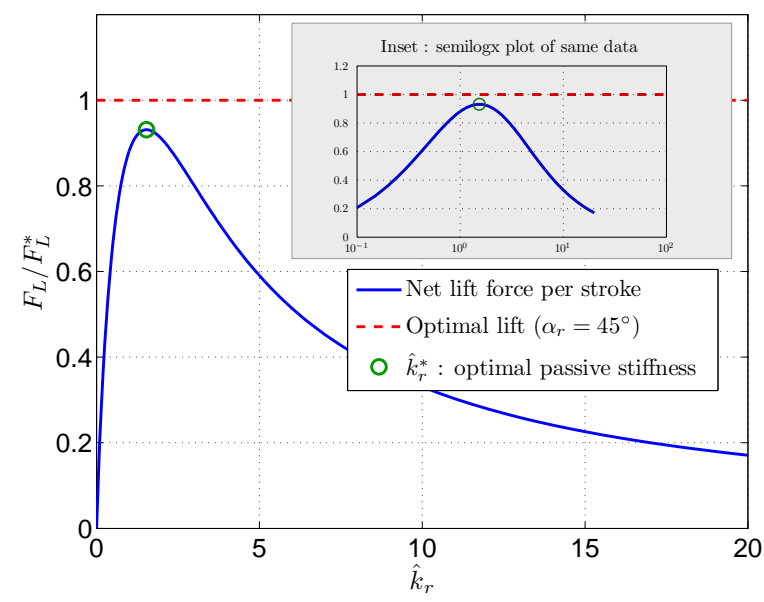

Figure 9. OPTIMAL PASSIVE STIFFNESS FOR LIFT.

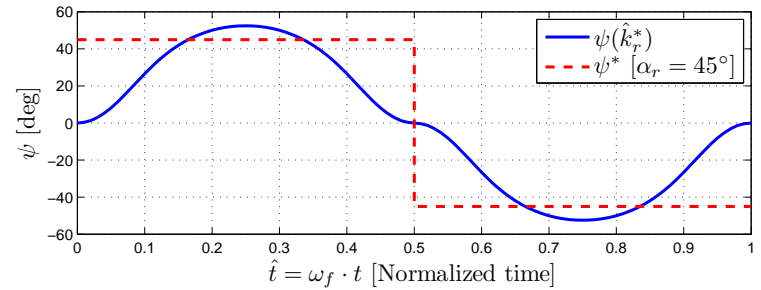

Figure 10. $\psi(t)$ WITH OPTIMAL $k_{r}$.

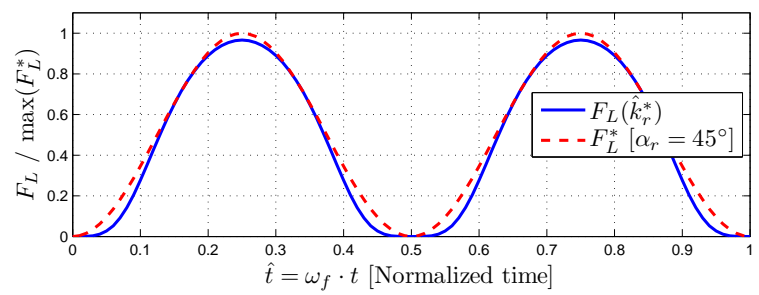

Figure 11. $F_{L}(t)$ WITH OPTIMAL $k_{r}$.

Figure 9 shows the time-averaged lift generated over a range of non-dimensionalized stiffnesses, $\hat{k}_{r}$. Here, $F_{L}$ is normalized by the optimal theoretical value that could be achieved if $\psi$ were controlled actively throughout a stroke, instead of being set by the wing impedance. From data in Figure 9, an optimal passive hinge results in 0.9311 times the lift of optimal, active control of $\psi$.

The non-dimensionalized optimal stiffness in Figure 9 is $\hat{k}_{r}=1.533$, and the actual stiffness for particular vehicle parameters scales as:

$$
k_{r}=\hat{k}_{r} \cdot \hat{\rho} \cdot\left(S_{w} R_{w}^{5}\right) \cdot \omega_{f}^{2} \phi_{\max }^{2}
$$

Here $\phi_{\max }=\pi / 3$ is the driven wing stroke amplitude, $\hat{\rho}$ is the density of the fluid, normalized by $\rho_{\text {air }}=$ $1.28\left(\mathrm{~kg} / \mathrm{m}^{3}\right)$, and $S_{w}$ is a non-dimensional shape factor for our particular, scalable wing shape:

$$
\begin{aligned}
S_{w} & =\frac{1}{R_{w}^{5}} \int_{r=0}^{R_{w}} c_{m}(r) c(r) r^{2} d r \\
& =0.00594
\end{aligned}
$$

From Equation 16, the optimal angle of attack for lift generation is just a constant value of $45^{\circ}$ through stroke, resulting a step function in $\psi(t)$, as shown in Figure 10. This figure also shows the passive motion of $\psi$ over the course of a single wingbeat when $k_{r}$ is tuned 
optimally, and Figure 11 compares $F_{L}(t)$ for the tuned, passive hinge versus for $\psi=45^{\circ}$. A similar plot comparing $F_{D}$ in both cases is shown in Figure 12. Here, $F_{D}$ is multiplied by $\operatorname{sgn}(\dot{\phi})$ to make it visually clear that the net forces in one wingbeat sum to zero.

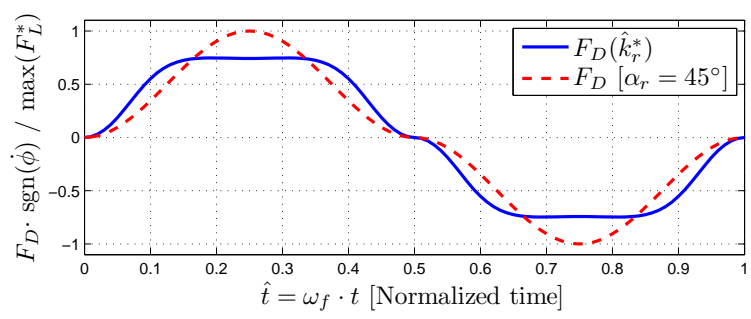

Figure 12. $F_{D} \cdot \operatorname{sgn}(\dot{\phi})$ WITH OPTIMAL $k_{r}$.

Varying the Offset Angle, $\psi_{o}$

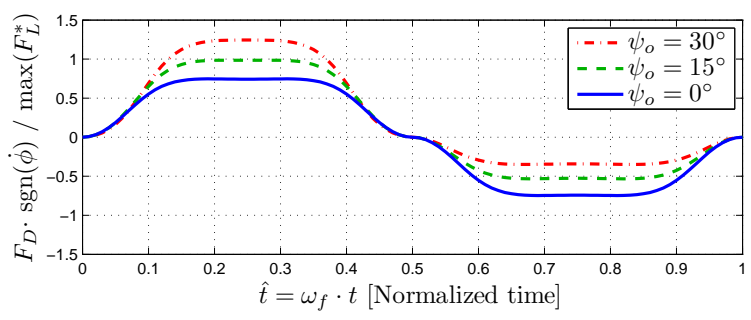

Figure 13. $F_{D}(t) \cdot \operatorname{sgn}(\dot{\phi})$ AS $\psi_{o}$ VARIES.

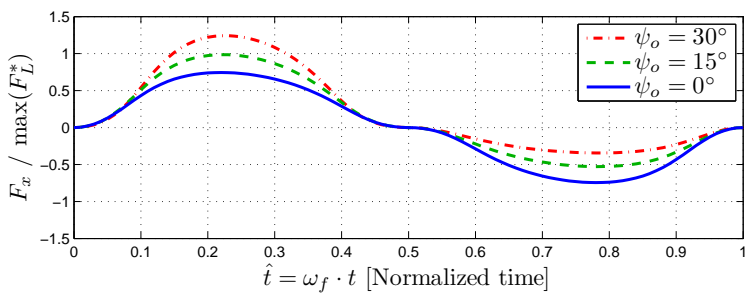

Figure 14. $F_{x}(t)$ AS $\psi_{o}$ VARIES.

Figures 13 and 14 show variations in $F_{D}$ and in $F_{x}$, respectively, over one wingbeat for different values of $\psi_{o}$. The magnitude of the force asymmetry produced can be visualized clearly in Figure 15. These data all correspond to the same value of $\hat{k}_{r}$. Note that although $F_{D}$ increases as the offset wing pitch angle increases, the magnitude of $F_{L}$ decreases. From Table 1, each vehicle produces enough lift to carry significantly more than its weight. $\left(\bar{F}_{L} / g_{z}=10,600 \mathrm{mg}\right.$, vs $m=6,000 \mathrm{mg}$ for the hummingbird scale design, for instance.) Since $60 \%$ of peak lift is adequate for hover for either model, we assume $\psi_{o}$ is kept less than $45^{\circ}$, where $F_{L} / F_{L}^{*} \approx 0.6$. At this value, $F_{D} / F_{L}^{*}$ is also approximately 0.6 , giving us our peak force (and acceleration) estimates in the $x$ direction in Table 1.

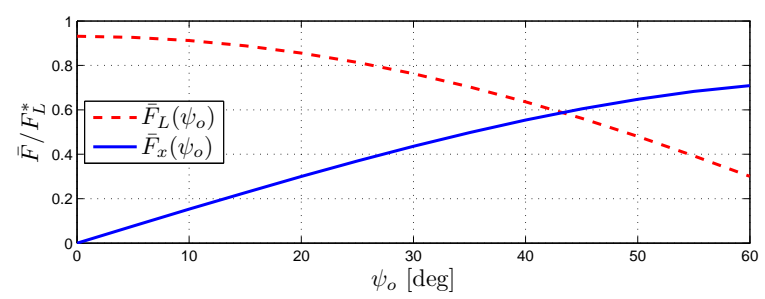

Figure 15. $F_{L}$ AND $F_{x}$ VS $\psi_{o}$.

Altitude control can be achieved by de-tuning both lefthand and righthand spring stiffnesses in unison, while asymmetries in these tunings can achieve roll torques. Similarly, forward flight and yaw turns can be controlled by adjusting the set-points of the left and right wings together or in opposition, respectively. Estimates for $\tau_{z}$ (yaw) and $\tau_{x}$ (roll) in Table 1 assume that the distance from the center of mass of the vehicle (to the wing root and then) to the $C O P$ on the wing is $\sim R_{w}$.

\section{DISCUSSION}

Our primary goal here is to demonstrate the range of control outputs that can be achieved by modifying only the passive impedance properties of a wing. However, we again emphasize that it is clearly possible - and will likely be optimal - to utilize both active modification of the power stroke (amplitudes, offsets, frequencies and waveforms of both the left and right wings) in conjunction with a passivity-based control approach, such as the one outlined here. We anticipate such a hybrid approach can maximize the range of control forces and torques possible while potentially increasing steady-state stability of hovering and constant-velocity flight, as well.

As a final comment, we note that while ignoring wing mass and inertia is a reasonable approximation for Diptera and other small insects, it becomes more questionable as wingspan increases [23]. Additionally, our model assumes laminar flow and a corresponding Reynolds number below 20,000 or so. The larger robot was specifically selected, with $R e \approx 20,000$, to be just at this desired limit, and $R e$ scales as $R_{w}^{2} \cdot \omega_{w}$. Thus, we caution the reader to take care in extending this analysis beyond the scale of a hummingbird-sized aircraft. 


\section{CONCLUSIONS}

We present a control approach where an appropriately set combination of a periodic stroke-angle waveform and a tuned, variable-impedance wing hinge can generate desired, time-averaged forces and torque to maneuver a small-scale, flapping-wing robot. Specifically, aerodynamic pressure forces on the wing are modulated indirectly by adjusting the set point and tension of a torsional spring element for wing pitch on each wing.

Using a quasi-static model, we find that the magnitudes of the forces that can be generated on a flappingwing MAV scale in proportion to $R_{w}^{4} \cdot \omega_{w}^{2}$, and we predict that for fly- to hummingbird- scale robots, accelerations on the order of 1-2 times gravity are feasible, even without exploiting non-quasistatic aerodynamics.

\section{REFERENCES}

[1] Raibert, M., Blankespoor, K., Nelson, G., Playter, R., and the BigDog Team, 2008. "Bigdog, the rough-terrain quadruped robot". Proceedings of the 17th World Congress, The International Federation of Automatic Control.

[2] Collins, S. H., Ruina, A., Tedrake, R., and Wisse, M., 2005. "Efficient bipedal robots based on passive-dynamic walkers". Science, 307, February 18, pp. 1082-1085.

[3] Wood, R., 2008. "The first takeoff of a biologically inspired at-scale robotic insect". IEEE Transactions on Robotics, 24(2), Apr, pp. 341347.

[4] Dudley, R., 2000. The Biomechanics of Insect Flight: Form, Function, Evolution. Princeton University Press.

[5] Dickinson, M. H., and Tu, M. S., 1997. "The function of Dipteran flight muscle". Comp. Biochem. Physiol., 116A, pp. 223-238.

[6] Taylor, G. K., 2001. "Mechanics and aerodynamics of insect flight control". Biol. Rev., 76, pp. 449-471.

[7] Dickinson, M., Lehmann, F.-O., and Gotz, K. G., 1993. "The active control of wing rotation by Drosophila”. J. Exp. Biol., 182, pp. 173-189.

[8] Bergou, A. J., Xu, S., and Wang, Z. J., 2007. "Passive wing pitch reversal in insect flight". J. Fluid Mech., 591, pp. 321-337.

[9] Schenato, L., Campolo, D., and Sastry, S., 2003. "Controllability issues in flapping flight for biomimetic micro aerial vehicles (mavs)". IEEE International Conference on Decision and Control, December.

[10] Hu, M., Wei, R., Shi, Z., and Zou, L., 2008. "Controllability issues for insect like flapping wing micro-air vehicle". In : Intelligent Control and Automation, 2008. WCICA 2008. 7th World Congress on, pp. 6365-6368.

[11] Ellington, C. P., 1984. "The aerodynamics of hovering insect flight I. the quasi-steady analysis". Philosophical Transactions of the Royal Society of London., 305(1122), pp. 1-15.

[12] Sane, S. P., and Dickinson, M. H., 2001. "The control of flight by a flapping wing: lift and drag production". Journal of experimental biology, 204, pp. 2607-2626.

[13] Osborne, M. F. M., 1951. "Aerodynamics of Flapping Flight with Application to Insects". J. Exp. Biol., 28(2), pp. 221-245.

[14] Dickinson, M. H., Lehmann, F.-O., and Sane, S. P., 1999. "Wing rotation and the aerodynamic basis of insect flight". Science, 284(5422), June, pp. 1954-60.

[15] Taylor, G., and Thomas, A., 2002. "Animal flight dynamics II. Longitudinal stability in flapping flight". Journal of Theoretical Biolgogy, 214, pp. 351-370.

[16] Dickson, W. B., and Dickinson, M. H., 2004. "The effect of advance ratio on the aerodynamics of revolving wings". Journal of Experimental Biology, 207, Aug, pp. 4269-4281.

[17] Young, John, Walker, M., S., Bomphrey, J., R., Taylor, K., G., Thomas, and R., A. L., 2009. "Details of Insect Wing Design and Deformation Enhance Aerodynamic Function and Flight Efficiency". Science, 325(5947), pp. 1549-1552.

[18] Sane, S., and Dickinson, M. H., 2002. "The aerodynamic effects of wing rotation and a revised quasi-steady model of flapping flight". J. Exp. Biol., 205, pp. 1087-1096.

[19] Wang, Z. J., Birch, J. M., and Dickinson, M. H., 2004. "Unsteady forces and flows in low Reynolds number hovering flight: two-dimensional computations vs robotic wing experiments". J. Exp. Biol., 207, pp. 449-460.

[20] Deng, X., Schenato, L., Wu, W. C., and Sastry, S. S., 2006. "Flapping flight for biomimetic robotic insects: Part I - System modeling”. IEEE Transactions on Robotics, 22(4), pp. 776-788.

[21] Altshuler, D. L., Dudley, R., and Ellington, C. P., 2004. "Aerodynamic forces of revolving hummingbird wings and wing models". J. Zool., 264, pp. 327-332.

[22] Hedrick, T. L., Cheng, B., and Deng, X., 2009. "Wingbeat time and the scaling of passive rotational damping in flapping flight". Science, 324, Ap, pp. 252-255.

[23] Dudley, and Robert, 2002. "Mechanisms and implications of animal flight maneuverability". Int. and Comp. Biol., 42(1), pp. 135-140. 